\title{
TEM Studies of the Oxidation of 316 Stainless Steel with In-Situ Proton Irradiation
}

\author{
Stephen S. Raiman, Peng Wang, Zhijie Jiao, Gary S. Was \\ University of Michigan: 2355 Bonisteel Boulevard, Ann Arbor, MI, 48109
}

The aggressive conditions inside light water reactors pose a risk to core internals as the conditions for aqueous corrosion are generally favorable due to the high temperature and concentration of oxidizing species. The addition of radiation has been shown to greatly accelerate the corrosion rate. Although experimental data is limited, order-of-magnitude increases in oxide thickness under irradiation have been observed[1-3]. The objective of this work is to observe the accelerating effect of irradiation on corrosion, and to examine the dual-layer oxide that forms on stainless steel.

316L stainless steel samples were irradiated while simultaneously exposed to simulated PWR conditions in a flowing corrosion cell with monitored water chemistry. The corrosion cell holds a $10 \mathrm{~mL}$ volume of water, and allows access for diagnostics. The 37- $\mu \mathrm{m}$-thick, dome-shaped samples serve as a "window" into the cell through which a $3.2 \mathrm{MeV}$ proton beam is passed, so the effect of radiation can be studied at the water-steel interface. The samples have been carefully designed to allow the proton beam to pass completely through the sample, while still serving as a barrier between the proton beam vacuum and the high-pressure, high temperature water in the corrosion cell. This design enables the incident radiation to influence the corrosion process in both the solid (displacement damage) as well as in the liquid (water radiolysis).

The sample was exposed to water with $3 \mathrm{ppm} \mathrm{H}_{2}$ and heated to $320^{\circ} \mathrm{C}$. Once at temperature, the sample was irradiated for 24 hours with a proton beam current of $10 \mu \mathrm{A} / \mathrm{cm}^{2}$ to a total dose of $2.5 \mathrm{dpa}$. A schematic of the experimental setup with images showing the irradiated area of the sample area are shown in figure 1.

FIB liftouts were taken from the irradiated and unirradiated areas of the sample, and examined by STEM, as shown in figure 2. The precipitated particles which make up the outer oxide are much smaller and more densely packed in the irradiated area when compared to the unirradiated area. The average thickness of the inner passive layer in the irradiated area is $70 \mathrm{~nm}$ compared to $140 \mathrm{~nm}$ in the unirradiated area.

EDS Line scans were taken over the irradiated and unirradiated oxides, and are shown in figure 3. The inner oxide of the irradiated area has a lower chromium to iron ratio than the inner layer of the unirradiated oxide.

\section{References}

1. Lapuerta, S., et al., The influence of relative humidity on iron corrosion under proton irradiation. Journal of Nuclear Materials, 2008. 375(1): p. 80-85.

2. Lewis, M.B. and J.D. Hunn, Investigations of ion radiation effects at metal/liquid interfaces. Journal of Nuclear Materials, 1999. 265(3): p. 325-330.

3. D. H. Bradhurst, P.J.S., P. M. Heuer, The Effects of Radiation and Oxygen on the Aqueous Oxidation of Zirconium and its Alloys at $290^{\circ} \mathrm{C}$. Journal of Nuclear Materials, 1972. 46: p. 53-76.

This work was supported by grants from the DOE-NEUP, grant number DE-AC07-05ID14517; and EDF, Contract No. 8610BVW-4300243004. 


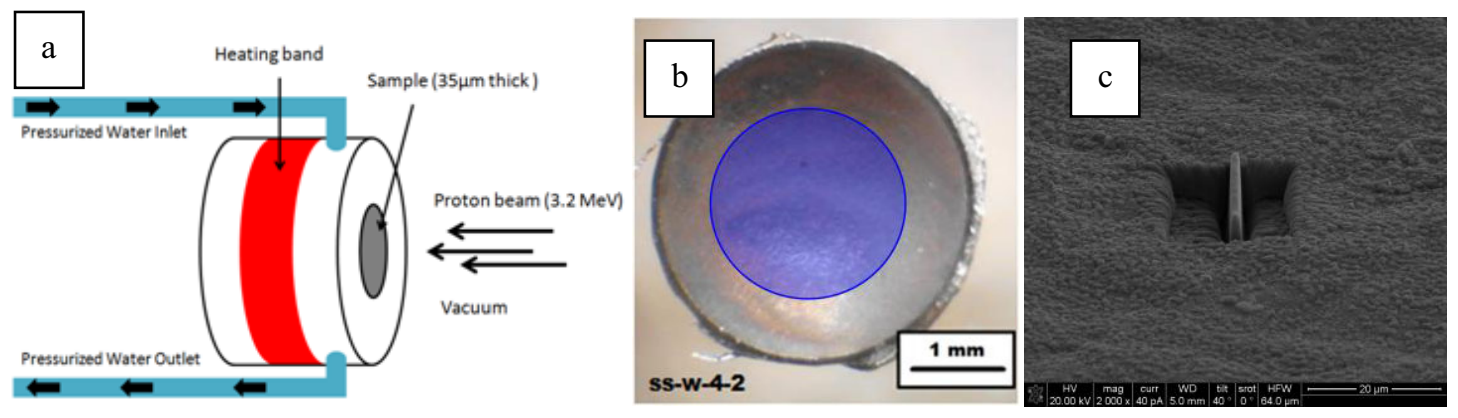

Figure 1. (a)A schematic of the corrosion cell (b)The water-side of the sample with the irradiated area shaded in blue, and the liftout locations circled in red (c) The oxidized sample surface in the irradiated area, with trenches dug around a liftout before extraction
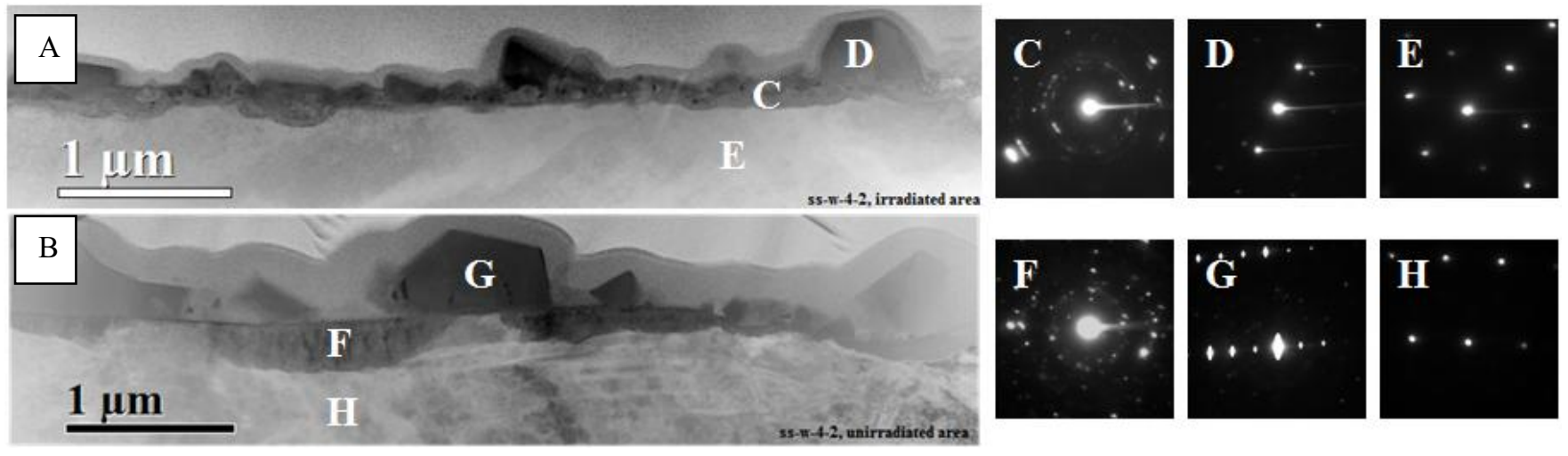

Figure 2. BF STEM images of the oxide cross-section from the (A)irradiated area and (B) unirradiated area. A dual-layer platinum coating is applied on top of each oxide. Diffraction patterns are taken from the irradiated area on the (C) outer oxide, (D) inner oxide, and (E) matrix; and on the unirradiated area on the $(\mathrm{F})$ outer oxide, $(\mathrm{G})$ inner oxide, and $(\mathrm{H})$ matrix. Patterns on all oxides are consistent with the spinel structure, and matrix patterns are consistent with stainless steel.
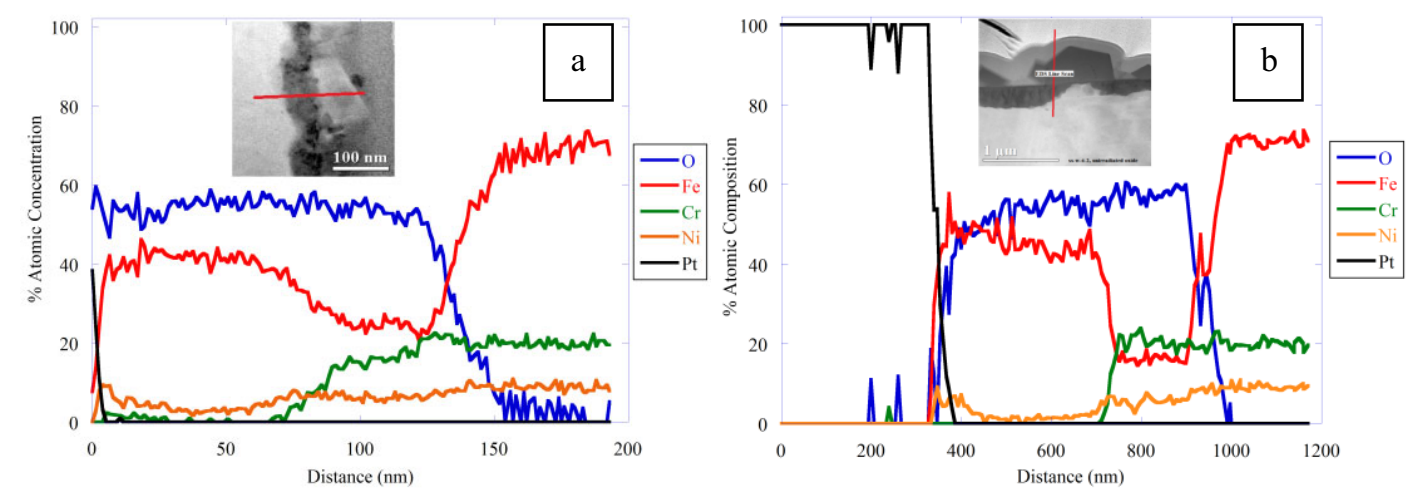

Figure 3. EDS scans across the oxide layers of the (a) irradiated and (b) unirradiated oxides. Chromium appears to be depleted in the irradiated inner oxide layer when compared to the unirradiated inner layer. 